\title{
Evolution from AGB to planetary nebula in the MSX survey ${ }^{\star} \star \star$
}

\author{
R. Ortiz ${ }^{1}$, S. Lorenz-Martins² ${ }^{2}$ W. J. Maciel ${ }^{3}$, and E. M. Rangel ${ }^{1}$ \\ ${ }^{1}$ Departamento de Física, UFES, Av. Fernando Ferrari 514, 29075-910, Vitória, ES, Brazil \\ e-mail: ortiz@cce.ufes.br \\ 2 GEMAC, Depto. de Astronomia, OV/UFRJ, Ladeira Pedro Antonio 43, 20080-090, Rio de Janeiro, RJ, Brazil \\ 3 Departamento de Astronomia, IAG/USP, Rua do Matão, 1226, Cidade Universitária, 05508-900, São Paulo, SP, Brazil
}

Received 6 March 2004 / Accepted 27 August 2004

\begin{abstract}
We investigate the evolution of oxygen- and carbon-rich AGB stars, post-AGB objects, and planetary nebulae using data collected mainly from the MSX catalogue. Magnitudes and colour indices are compared with those calculated from a grid of synthetic spectra that describe the post-AGB evolution beginning at the onset of the superwind. We find that carbon stars and $\mathrm{OH} / \mathrm{IR}$ objects form two distinct sequences in the $(K-[8.3]) \times([8.3]-[14.7]) \mathrm{MSX}$ colour diagram. OH/IR objects are distributed in two groups: the bluest ones are crowded near [14.7]-[21.3] $\simeq 1$ and [8.3]-[14.7] $\simeq 2$, and a second, redder group is spread over a large area in the diagram, where post-AGB objects and planetary nebulae are also found. High massloss rate OH/IR objects, post-AGB stars, and planetary nebulae share the same region in the $(K-[8.3]) \times([8.3]-[14.7])$ and [14.7]-[21.3] $\times([8.3]-[14.7])$ colour-colour diagrams. This region in the diagram is clearly separated from a bluer one where most $\mathrm{OH} / \mathrm{IR}$ stars are found. We use a grid of models of post-AGB evolution, which are compared with the data. The gap in the colour-colour diagrams is interpreted as the result of the rapid trajectory in the diagram of the stars that have just left the AGB.
\end{abstract}

Key words. stars: AGB and post-AGB - stars: circumstellar matter - stars: evolution - ISM: planetary nebulae: general infrared: stars

\section{Introduction}

AGB and post-AGB evolution have lately become better understood, thanks to numerous surveys carried out by different groups at several wavelengths, from radio (e.g. CO rotational transitions, $\mathrm{OH}$ maser emission at $18 \mathrm{~cm}$ ) to infrared. The present knowledge of stellar evolution in the AGB (Iben Jr. 1995) states that the more massive stars in this phase may undergo a third dredge-up episode, which enriches their convective envelope with carbon that will eventually reach the atmosphere resulting in a "carbon-rich star". However, if the star does not experience the third dredge-up, or if its evolution has not reached this point, its atmosphere remains oxygen-rich. Therefore the chemical composition of the atmosphere in the AGB is dependent on both the initial mass and the evolutionary phase of the star. In any case, when the mass loss rate increases, which is believed to happen at the end of the AGB, an optically thick circumstellar dust shell (CDS) develops, making the star invisible at visual wavelengths. The star then climbs up the AGB becoming very luminous $\left(L_{\mathrm{bol}} \simeq 10^{3}-10^{6} L_{\odot}\right)$ especially in the infrared, most of its flux being emitted by cold and warm grains ( $T_{\text {dust }} \sim 300-900 \mathrm{~K}$, depending on the chemical

\footnotetext{
^ Based on results obtained by the MSX survey.

$\star \star$ Tables 1 to 3 are only available in electronic form at the CDS via anonymous ftp to cdsarc.u-strasbg.fr $(130.79 .128 .5)$ or via http://cdsweb.u-strasbg. fr/cgi-bin/qcat? J/A+A/431/565
}

composition) in the CDS. This twofold scenario (large infrared flux and low colour temperature) makes AGB stars, together with young stellar objects, the main body of detections in infrared surveys. They are detected at large distances, such as several kiloparsecs, as far as the galactic bulge and beyond. In addition to infrared continuum emission, AGB stars may exhibit a variety of molecular lines including $\mathrm{CO}, \mathrm{HCN}$ (in carbon-rich); $\mathrm{OH}, \mathrm{SiO}$, and $\mathrm{H}_{2} \mathrm{O}$ (in oxygen-rich stars). The emission originates in the circumstellar shell and, together with some features observed in the infrared, it represents the chemical $(\mathrm{C} / \mathrm{O})$ signature of the atmosphere of the star. Hydroxyl maser emission at $18 \mathrm{~cm}$ is quite common and its $1612 \mathrm{MHz}$ component becomes particularly strong in optically thick shells; this is widely known as the $\mathrm{OH} / \mathrm{IR}$ phenomenon.

After the AGB phase the star follows a horizontal track in the Hertzprung-Russell diagram (Schönberner 1981, 1983). The convective envelope of the star is expelled and its hot core revealed. This process takes about $10^{3} \sim 10^{4}$ years before the luminosity of the core decreases and the star moves towards the white dwarf cooling track. Immediately after the envelope is expelled a reflection nebula appears around the star. Eventually a small HII region appears in the inner part of the nebula followed by metallic emission lines of increasing ionization potential in the spectrum. This hybrid object, which still shows some characteristics of the AGB phase like intense infrared continuum and molecular bands, is often called "post-AGB" 
star or "proto planetary nebula". Here we prefer the name "transition object", without making distinctions in the true evolutionary status during its horizontal trajectory after the end of the AGB. The study of these objects has in fact shown that they are evolved objects, with chemical abundances and metallicities that resemble those of AGB stars, showing enhanced s-process and $\mathrm{CN}$ abundances. About one to two hundred candidates have been suggested, observed and confirmed as transition objects, so far.

Van der Veen \& Habing (1988, hereafter VH) studied the distribution of different kinds of AGB star in the [12-25] $\times$ [25-60] colour-colour diagram. The diagram was divided into regions, each corresponding to one type of object. Regions I through IIIb (see Fig. 5b in VH) contain the stars evolving from O-rich Miras to OH/IR stars. Region IV mainly consists of transition objects, and region $\mathrm{V}$ of planetary nebulae. The release of the MSX point source catalogue (Egan et al. 1997) has offered a new opportunity to study the AGB to PN evolution in mid-infrared colour-colour diagrams. The main scope of this work is to verify whether the AGB to PN evolution in MSX colour-colour diagrams follows a pattern similar to what was found by $\mathrm{VH}$ using the IRAS colour-colour diagram. We make use of samples containing OH/IR objects, C-rich AGB stars, transition objects, and planetary nebulae, to study how these objects are spread in different MSX (and nearinfrared) colour-colour diagrams. We also compare the distributions above with predictions of synthetic theoretical models of post-AGB evolution. The scheme of this paper is as follows: in Sect. 2 we present an overview of the database used in this work; in Sect. 3 colour-colour diagrams are discussed; Sect. 4 contains a discussion about the nature of the transition objects; Sect. 5 is devoted to the comparison between theoretical models for post-AGB evolution and the data; and in Sect. 6 we present our conclusions.

\section{Surveys of evolved objects: The data}

The major mid-infrared database used in this work is the point source catalogue of the MSX (Midcourse Space Experiment; Egan et al. 1997) project. The main scope of the MSX survey was to cover the whole galactic plane at $|b|<5^{\circ}$ in the infrared, between $4.2 \mu \mathrm{m}<\lambda<27 \mu \mathrm{m}$. This region had been formerly covered by the IRAS satellite, however due to various technical limitations, the IRAS mission did not have spatial resolution suited to the crowdedness of the galactic plane. On the other hand, MSX combines better spatial resolution (18.3 arcsec), with sensitivity and wide wavelength coverage which allows one to resolve red giant stars in moderately crowded regions in the galactic plane. Version 1.0 of the catalogue, which is used in this work, contains 323052 sources. All MSX flux densities have been converted into magnitudes according to the Explanatory Guide (Egan et al. 1999). The zero-magnitude flux densities are the following: 58.49, 26.51, 18.29, and 8.80 Jansky, corresponding to bands A, C, D, and E, respectively. These zero-points were calculated relative to a blackbody with $10000 \mathrm{~K}$ temperature. The isophotal wavelengths of these bands are: $8.28 \mu \mathrm{m}$ (band A), $12.13 \mu \mathrm{m}$ (band C), $14.65 \mu \mathrm{m}$ (band D), and $21.34 \mu \mathrm{m}$ (band E).
Bands B1 and B2, centered near $\lambda=4.3 \mu \mathrm{m}$ were discarded in this work due to their poor sensitivity.

A large number of molecular surveys has been carried out towards several regions of the Galaxy. $\mathrm{OH}$ surveys are especially suited to detect OH/IR stars. Benson et al. (1990) compiled a catalogue of $\mathrm{OH}$ observations made by several authors. More recently, $\mathrm{OH}$ observations have been carried out towards the bulge (Sjouwerman 1998; Lindqvist 1998) and the galactic plane (Sevenster 1997a,b, 2001), among others. Carbon-rich stars can be detected at microwave frequencies, especially by observing the $\mathrm{CO}$ and $\mathrm{HCN}$ transitions. In this work we use the list of microwave observations of these two molecules made by Loup et al. (1993), comprising 213 stars identified in the literature as carbon-rich stars. The OH/IR objects are taken from the galactic plane $\mathrm{OH}$ maser surveys of Sevenster cited above, amounting to 766 objects. The sample of transition objects comprises 106 objects taken from several papers in the literature, consisting of a wide range of evolutionary stages, ranging from AGB stars showing evidence of a superwind to newborn planetary nebulae. The catalogue of planetary nebulae used in this study is that of Kimeswenger (2001), which contains 995 objects in the southern hemisphere.

The sample of carbon stars mentioned above (Loup et al. 1993) was cross-correlated with the MSX catalogue of point sources. One must keep in mind that the compilation of Loup et al. contains stars distributed in all directions, observed with different radio telescopes and thus the spatial resolution changes from source to source. We chose to search the MSX catalogue within 10 arcsec from the radio positions as Loup et al. consider this figure representative for objects that match an IRAS source. This is much larger than the astrometric accuracy claimed by the MSX team ( 2 arcsec) but still smaller than the spatial resolution of the MSX survey $\left(18.3^{\prime \prime}\right)$. Near-infrared counterparts were mainly taken from the literature (Guglielmo et al. 1993, 1997, 1998; Gezari et al. 1987). These papers provide the $K-L^{\prime}$ colour, which is an excellent estimator of the optical depth of the circumstellar shell (Lépine et al. 1995; Epchtein et al. 1987). When the near-infrared photometry was not found in the references above, we looked for a 2MASS counterpart within 3 arcsec of the radio position. The search above resulted in 45 MSX counterparts (Table 1), all of them with a counterpart in the near-infrared, taken either from the literature or from the 2MASS point-source catalogue.

The list of OH maser sources by Sevenster (1997a,b, 2001) was obtained using both the ATCA and the VLA, which makes these data more homogeneous concerning astrometric accuracy and flux calibration, for instance, than the present sample of carbon stars. Infrared counterparts of the 766 objects detected in these OH surveys have been studied by Sevenster (2002a,b), who found 494 MSX and 194 2MASS objects within 5" and $3^{\prime \prime}$ of the radio position, respectively. In this work, when cross-correlation involving $\mathrm{OH}, \mathrm{MSX}$ and 2MASS is needed, we assume the identifications provided by Sevenster (2002a). To the 194 2MASS counterparts identified by Sevenster we added 28 more, taken from various sources in the literature, amounting to a total of 222 objects.

Since we intend to study how AGB stars evolve into planetary nebulae, it is important to compile a sample that contains a 


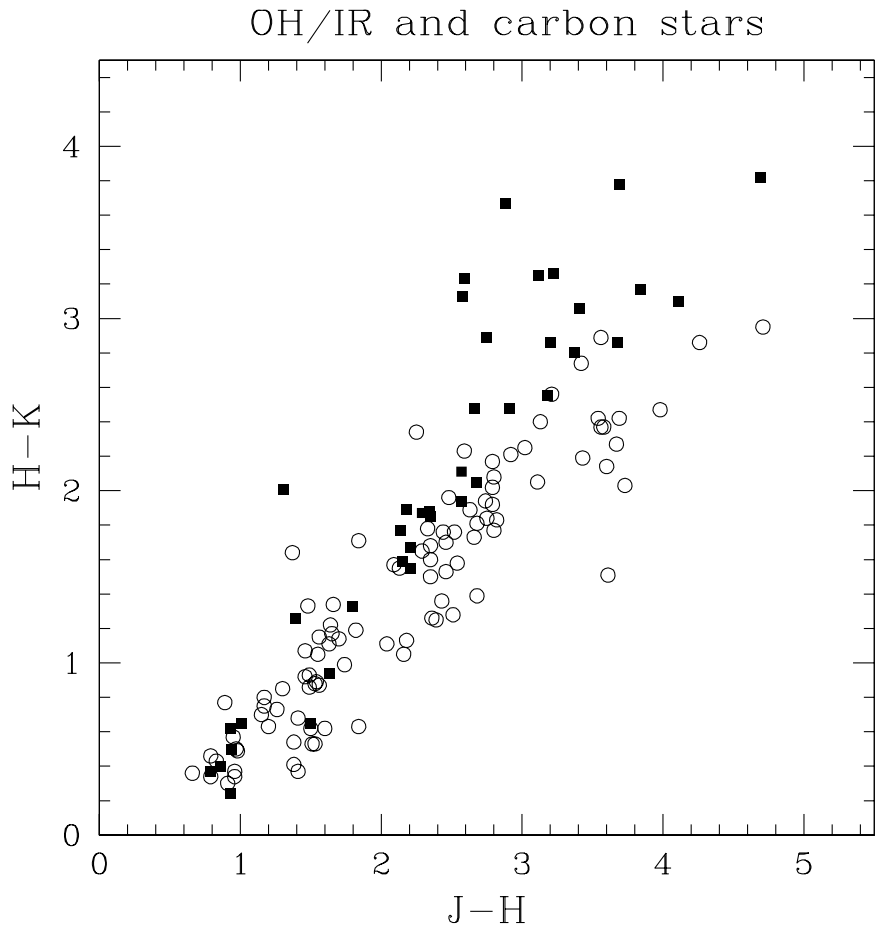

Fig. 1. $(J-H) \times(H-K)$ colour-colour diagram of C-rich (squares) and $\mathrm{OH} / \mathrm{IR}$ objects (open circles), with a MSX counterpart. The nearinfrared photometry was extracted from the literature and the 2MASS database.

wide range of characteristics, especially the optical depth of the envelope, which depends mainly on the mass-loss rate. In this case, near-infrared colour indices are more appropriate than indices involving the near-and-mid-infrared, because the latter are often influenced by numerous molecular features like $\mathrm{SiO}$. Figure 1 shows the $(J-H) \times(H-K)$ colour-colour diagram for carbon-rich stars and OH/IR objects that have a MSX counterpart. One can see that the present sample includes stars in a wide range of colours up to $J-H=4.6$. Therefore we can assume that our sample also contains stars in a wide range of optical depths and mass-loss rates.

The present sample of transition objects amounts to 106 stars, all of them having an IRAS counterpart and $|b|<5^{\circ}$, which is the area surveyed by MSX. It was compiled from numerous sources in the literature. Similarly to the carbon stars, we searched for MSX counterparts within $10^{\prime \prime}$ of the IRAS position and as a result we obtained 69 sources (Table 2). Most of them $(64$ objects $=93 \%)$ have a near-infrared counterpart either in the literature or taken from the 2MASS database.

Among the 995 planetary nebulae in the list of Kimeswenger, 606 have $|b|<5^{\circ}$, the area covered by MSX. We searched for MSX counterparts within 10" of the catalogue position, and found 214 objects. Among these, 162 have diameters $\theta<18^{\prime \prime}$, which is the spatial resolution of the MSX survey, listed in the catalogue by Acker et al. (1992). These nebulae can be considered as point sources and have been selected for study (Table 3 ). Among these 214 objects, 89 nebulae have reliable $K$ magnitudes, whereas 73 have their magnitudes listed as lower limits.
Table 4 collects the figures concerning the several source catalogues used in this work, their sky coverage, number of MSX counterparts found, near-infrared counterparts, etc. All objects selected for study in this paper have an MSX counterpart found according to the criteria described above.

\section{Post-AGB evolution in colour-colour diagrams}

Colour-colour diagrams have often been used to discriminate among different classes of AGB stars, especially concerning their chemical features. After the IRAS mission, when a large number of low-resolution spectra of AGB stars was taken, it became clear that the silicate feature at $9.7 \mu \mathrm{m}$ is the more prominent band in the mid-infrared in most cases. Spectra were classified as having this feature in emission (type $2 \mathrm{n}$ ), or in absorption (3n). Sources showing the $\mathrm{SiC}$ feature at $11.3 \mu \mathrm{m}$, which is characteristic of carbon-rich AGB stars, are classified as type $4 \mathrm{n}$. This feature is much less intense than the silicate one. As a result, the distribution of energy of carbon-rich stars in the mid-infrared is close to blackbody. On the other hand, the silicate feature at $9.7 \mu \mathrm{m}$ can affect the IRAS [12] magnitude significantly.

Epchtein et al. (1987) studied a sample containing different kinds of stars found during the "Valinhos" survey and studied how the IRAS magnitudes are affected by the SiC and $\mathrm{SiO}$ features. According to the authors, stars classified in the IRAS-LRS catalogue as $4 \mathrm{n}$ are all distributed in a region in the $(K-L) \times[12]-[25]$ which is close to the blackbody line. In this diagram, the $(K-L)$ colour is interpreted as a sequence of increasing mass-loss rate or optical depth of the circumstellar dust shell. The silicate feature at $9.7 \mu \mathrm{m}$ can appear either in emission or absorption, depending on the optical depth of the envelope. It is located well within the IRAS-[12] and the MSX-A band ranges. On the other hand, a much weaker and broader silicate feature exists at $18 \mu \mathrm{m}$, which generally appears in emission. This feature affects little the IRAS-[25] magnitude, whereas the MSX photometric bands are not affected at all. Consequently, the [12]-[25] colour index depends strongly on the strength of the silicate dust feature at $9.7 \mu \mathrm{m}$. When in emission (type $2 \mathrm{n}$ sources), the $9.7 \mu \mathrm{m}$ feature increases the star's flux in the [12] band, which makes this magnitude smaller. On the other hand, when in absorption (type $3 \mathrm{n}$ sources), this feature causes the [12] magnitude to increase. Although the [25] magnitude may also change with optical depth, the [12]-[25] colour index is expected to be larger for type $3 \mathrm{n}$ stars, which are those that exhibit larger mass-loss rates (the $\mathrm{OH} / \mathrm{IR}$ stars). In short, they found that O-rich stars appear separated from C-rich stars in the $(K-L) \times[12]-[25]$ diagram, and this is caused by the strength of the silicate feature at $9.7 \mu \mathrm{m}$.

Besides Epchtein et al., many papers suggest colour-colour diagrams as a tool to discriminate carbon from oxygen-rich stars. For instance, Van Loon et al. $(1997,1998)$ used the $K-[12] \times(H-K)$ colour diagram and van der Veen \& Habing (1988) placed carbon stars in sector VIa and VII of their [12]-[25] $\times$ [25]-[60] colour-colour diagram. Whatever the colour-colour diagram used, it generally contains the [12] band or any other band that includes the $9.7 \mu \mathrm{m}$ feature, as 
Table 4. Summary of the database. The number of objects in the last three columns refers to: (1) in the original reference; (2) with an MSX counterpart; (3) with an MSX and near-infrared counterpart. All objects selected for study in this paper have a counterpart in the MSX survey, which covered the whole galactic plane restricted to $|b|<5^{\circ}$.

\begin{tabular}{cccccc}
\hline \hline Object & Reference to the & Sky coverage & \multicolumn{3}{c}{ Number of objects } \\
& original database & & $(1)$ & $(2)$ & $(3)$ \\
\hline OH/IR & Sevenster (1997a,b, 2001) & $|l|<45^{\circ},|b|<3^{\circ}$ & 766 & 494 & 222 \\
C-rich stars & Loup et al. (1993) & all-sky & 213 & 45 & 45 \\
transition objects & several & $|b|<5^{\circ}$ & 106 & 69 & 64 \\
planetary nebulae & Kimeswenger (2001) & $\delta<+2^{\circ}$ & 995 & 214 & 86 \\
\hline
\end{tabular}

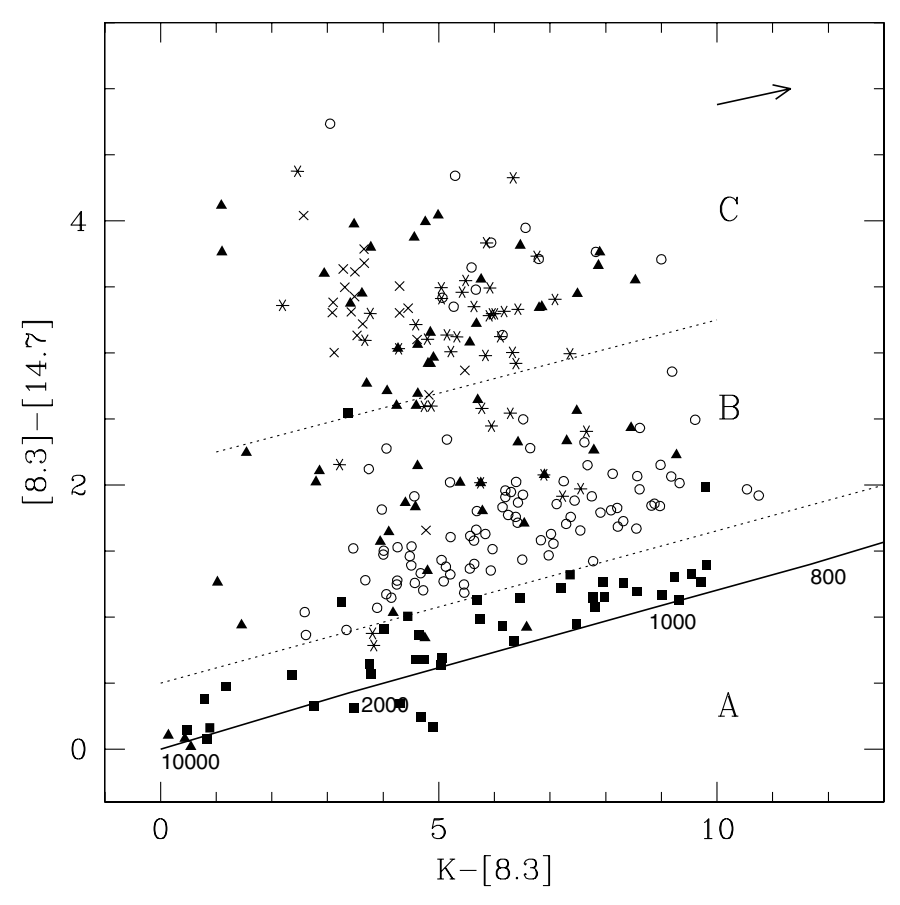

Fig. 2. MSX $-K$ colour-colour diagram showing carbon-rich stars, $\mathrm{OH} / \mathrm{IR}$ objects, transition objects, and planetary nebulae. The symbols represent: carbon-rich stars (squares), OH/IR objects (open circles), transition objects (triangles), and planetary nebulae (asterisks for well determined $K$ measurements and crosses for lower limits of $K$ ). The continuous line is the blackbody temperature sequence, and the two dotted lines separate regions A, B and C in the diagram. The arrow in the upper right corner represents the reddening for $A_{\mathrm{V}}=20 \mathrm{mag}$.

the MSX A band does. In the following, we will discuss where evolved objects are located in colour-colour diagrams.

Figure 2 shows a colour-colour diagram that is suitable for discriminating carbon stars from other evolved objects. The lower dotted line limits region A, where carbon stars are found and oxygen-rich stars are absent. The former appear to follow the blackbody line down to $T \simeq 900 \mathrm{~K}$. OH/IR stars are distinguished from carbon-rich objects especially by the [8.3]-[14.7] colour index, as discussed before in this section, consequently they are found above the carbon stars in this diagram. Objects which show lower mass-loss rates are distributed predominantly in region $\mathrm{B}$, while those that have higher values are mostly in region $\mathrm{C}$, which corresponds approximately to the upper-right region of Fig. 3, as we will eventually discuss.
Regions $\mathrm{B}$ and $\mathrm{C}$ are separated by a gap that runs along the upper dotted line in Fig. 2. This gap was formerly reported by Sevenster (2002a) in her [8]-[12] and [15]-[21] histograms and was interpreted as a separation between late-OH/IR and transition objects. As she points out, $\mathrm{OH} / \mathrm{IR}$ stars are mostly located in the third quadrant of the [8]-[12] $\times[15]-[21]$ colour-colour diagram, which corresponds to region B in our Fig. 2, and the lower-left corner in Fig. 3. According to Sevenster, the redder $\mathrm{OH} / \mathrm{IR}$ objects are mainly non-variable, post-AGB stars, which corresponds to region $\mathrm{C}$ of our diagram. The transition objects selected in this paper are found spread out in the three regions: there are 5, 24 and 27 objects in regions $\mathrm{A}, \mathrm{B}$ and $\mathrm{C}$, respectively. The proportion of planetary nebulae in the three regions follows approximately the same distribution. The figures shown above suggest that this diagram alone does not provide a clear discrimination between the redder $\mathrm{OH} / \mathrm{IR}$ objects, transition objects, and planetary nebulae, but it does provide a fine distinction between $\mathrm{C}$-rich and $\mathrm{OH} / \mathrm{IR}$ stars.

We looked for information on the five transition objects that are located in region $\mathrm{A}$, and we found that two of them are confirmed carbon-rich stars, two stars seem to be oxygenrich, and one is undetermined. MSX5C G290.0075+01.2915 = IRAS $11064-5842=$ V382 Car is a yellow (G4 0-I) supergiant which is listed in the list of Oudmaijer et al. (1992) as a star showing strong infrared excess. Its infrared spectrum shows several features between 6 and 9 micron, characteristic of stretching and bending vibrations of polycyclic aromatic hydrocarbons (PAH). MSX5CG 012.2187+04.9223 = IRAS 17514-1555 was suggested to be a transition object by $\mathrm{Hu}$ et al. (1993), and has its optical spectrum classified as WC 11 (Pena et al. 2003). The two oxygen-rich objects are MSX5CG 240.6415+03.0726 (=IRAS 07547-2244 = 11 Pup) and MSX5CG 015.3218-04.2673 (=IRAS 183131738 = MWC939). The former one has F7/F8 II spectral type and its $\mathrm{C} / \mathrm{O}$ abundance ratio is 0.28 (Luck \& Webfer 1995). It was extracted from the list of supergiant stars with infrared excesses compiled by Oudmaijer. The second oxygenrich star is of Be type (Vijapurkar et al. 1998) and shows the silicate feature at $\lambda=9.8 \mu \mathrm{m}$, of the SiO. The fifth object, MSX5CG 292.9528+01.8774 (=o Cen), for which we did not find information on its $\mathrm{C} / \mathrm{O}$ ratio, is a $\mathrm{G} 0$ Ia supergiant with infrared excess (Oudmaijer et al. 1992). These five objects have been selected by their optical characteristics (low gravity, early or intermediate spectral type), some of them 


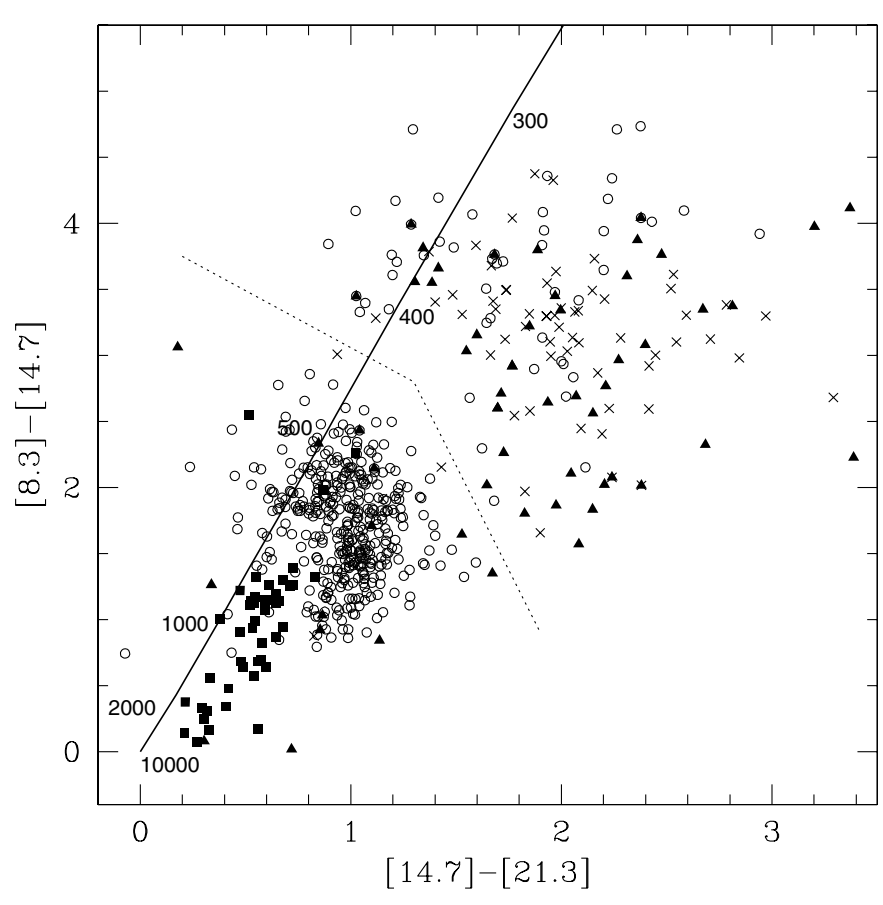

Fig. 3. MSX colour-colour diagram for carbon-rich stars, OH/IR objects, transition objects, and planetary nebulae. The symbols used are the same as those in Fig. 2 and the continuous line represents the blackbody temperature sequence.

associated with peculiar infrared properties (infrared excess). Their location in the $K-[8.3] \times[8.3]-[14.7]$ colour-colour diagram near the blackbody line indicates that they have no significant [8.3]-[14.7] infrared excess, else they would be in regions $\mathrm{B}$ or $\mathrm{C}$. We must recall that the stars extracted from the list of Oudmaijer were selected according to the criteria: IRAS [12]-[25] $>0.4$ and [25]-[60] $>0.3$, which might result in stars with colour temperatures in some cases as high as $\sim 1000 \mathrm{~K}$. On the other hand, the transition objects located in regions $\mathrm{B}$ and $\mathrm{C}$ have much lower colour temperatures. As we discuss in Sect. 5, the tracks that represent the stellar evolution beyond the AGB phase show that the MSX colour temperatures remain low long after the star leaves the AGB. Therefore it is possible that the few transition objects found in region A are not transition objects, even though they are considered "suspect" in the literature.

The behaviour of extinction in the MSX wavelength range is a little controversial, since it strongly depends on the chemical composition of the absorbing matter. In this work we consider the extinction curves given by Cox (2000) and $\mathrm{Li}$ $\&$ Greenberg (1997), and we have adopted their average, as follows: $A_{\mathrm{A}} / A_{\mathrm{V}}=0.024(\lambda=8.3 \mu \mathrm{m}) ; A_{\mathrm{C}} / A_{\mathrm{V}}=0.022$ $(\lambda=12.1 \mu \mathrm{m}) ; A_{\mathrm{D}} / A_{\mathrm{V}}=0.018(\lambda=14.7 \mu \mathrm{m}) ;$ and $A_{\mathrm{E}} / A_{\mathrm{V}}=$ $0.023(\lambda=21.3 \mu \mathrm{m})$. It is interesting to observe that the [14.7]-[21.3] colour becomes bluer with increasing extinction. Lumsden et al. (2002) found similar results, based on other references to extinction curves. Considering the discussion above, and the fact that the objects in this study are mostly a few kiloparsecs away, we can state that all pure MSX colours are little affected by reddening. The $K-[8.3]$ colour is just 0.66 magnitude reddened for $A_{\mathrm{V}}=10$ and is the most affected colour in this study (see Fig. 2), however this does not affect the classification of the sources in the regions $\mathrm{A}, \mathrm{B}$, and $\mathrm{C}$, since the reddening vector is almost parallel to the lines that separate these regions.

Figure 3 shows the various classes of evolved objects in the [8.3]-[14.7] $\times[14.7]-[21.3]$ colour-colour diagram. This diagram corresponds approximately to the [8]-[12] $\times[15]-[21]$ of Sevenster (2002a), her Fig. 3. The blackbody line is drawn in, together with the corresponding colour temperatures. We can define a region in the diagram where carbon stars are located, which is near the blackbody line at $T=800 \sim 2000 \mathrm{~K}$. These stars show a range of near-infrared colours as wide as $\mathrm{OH} / \mathrm{IR}$ objects do (see Fig. 1); however, at mid-infrared wavelengths the colour temperatures of carbon stars are higher. Near the region occupied by C-stars, but in a slightly lower temperature range $(T=800 \sim 1000 \mathrm{~K})$, a few OH/IR objects are also observed; however, the majority of these shows temperatures in the range $T=500 \sim 800 \mathrm{~K}$. As we shall discuss in Sect. 5, these $\mathrm{OH} / \mathrm{IR}$ objects probably are oxygen-rich AGB stars. A second, distinct group of $\mathrm{OH} / \mathrm{IR}$ objects is spread over a large area in the diagram, to the upper right of the dotted line, together with transition objects and planetary nebulae. The latter group of objects is separated from the first by a gap, which is indicated in Fig. 3 by the dotted line; it corresponds to the dips seen in the MSX colour histograms drawn by Sevenster. Because the definitions of magnitudes used in this paper are different from those used by Sevenster the gaps do not have the same colour indices as the dips. In this diagram, the OH/IR objects to the upper right of the dotted line fall in the same region as transition objects and planetary nebulae. These $\mathrm{OH} / \mathrm{IR}$ objects might be either stars close to leaving the AGB or transition objects with oxygen-rich envelopes.

The gap that runs along the dotted line in Fig. 3 is not a bias effect, but results from the rapid evolution of the stars as they cross it. Below we discuss this statement, which will be strengthened in Sect. 5, where we discuss results of theoretical models. As we remark in Sect. 2, the samples of OH/IR objects and C-rich stars both contain objects in a wide range of colour indices, which are indicative of a large variety of massloss rates and optical depths as well. These two parameters also depend also on the evolutionary stage of the star in the AGB phase. Lépine et al. (1995) and Epchtein et al. (1990) showed that $\mathrm{OH} / \mathrm{IR}$ and $\mathrm{C}$-rich stars in the various evolutionary stages are smoothly distributed along sequences in nearinfrared colour-colour diagrams. Therefore, since the stars in our samples are also smoothly distributed in colour (Fig. 1), we conclude that these two samples contain stars smoothly distributed concerning mass-loss rates, optical depths of the envelopes, and evolutionary stages.

At this point, a word must be said on the evolution of carbon-rich AGB stars in the colour-colour diagrams discussed above. As one can see in Fig. 3, there are no carbonrich stars on the upper right side of the dotted line, where numerous $\mathrm{OH} / \mathrm{IR}$ objects coincide with transition objects and planetary nebulae. This absence deserves an explanation, since carbon-rich stars are the result of the evolution of oxygen-rich AGB stars in the thermal-pulse phase, where the abundance of carbon exceeds that of oxygen. Therefore, it seems that 
carbon-rich AGB stars should coincide with OH/IR stars: both classes show high mass-loss rates, they are both in the thermalpulse phase and have optically thick envelopes. However, even the redder carbon-rich stars have higher colour temperatures than OH/IR stars. Here we discuss the reasons for this discrepancy. Ordinary carbon-rich stars have their spectral energy distribution (SED) maximum near 1-2 $\mu \mathrm{m}$. Despite the maximum shifting to longer wavelengths in the case of very red carbon-rich stars (like IRC +10216 , for example), its position is still located at wavelengths near to or shorter than $8 \mu \mathrm{m}$. The carbon-rich stars that one would expect to find in the upper right corner of MSX colour-colour diagrams are the extreme carbon stars (e.g. AFGL 3068), which have maxima near $\lambda=10 \mu \mathrm{m}$. However, even these extreme examples have moderate colour temperatures, about $T=2000-2500 \mathrm{~K}$. Thus, even the extreme carbon stars have temperatures which put these stars at the left bottom corner of Fig. 3. On the other hand, carbon-rich transition objects can show large MSX colour indices, because their SEDs are double peaked: they exhibit a maximum near $\lambda=1 \mu \mathrm{m}$, which originates from the central star, and a second (broad) maximum near $\lambda=10-20 \mu \mathrm{m}$. This second maximum accounts for the low MSX colour temperatures of these objects.

Both colour-colour diagrams discussed above were formerly proposed by Lumsden et al. (2002) in a paper where various types of young objects such as compact HII regions, massive young stellar objects, Herbig Ae/Be stars, are also discussed. Most of the results found in this work confirm their previous conclusions, but here we outline a few differences. As one can see in Fig. 1, the sample of carbon-rich stars contains a significant number of objects with optically thick circumstellar envelopes, whereas there are few of them in the work of Lumsden and his colleagues. The sample of carbon stars used in this work is based on the compilation by Loup et al. (1993), which is a collection of radio observations of the $\mathrm{CO}$ and HCN molecules. This list does not have an optical bias, which would select only stars with optically thin envelopes. On the other hand, Lumsden et al. (2002) remark that most of the carbon stars used in their work have been optically selected, although they include in their study a few heavily embedded carbon stars. Another important difference between our [14.7]-[21.3] $\times[8.3]-[14.7]$ diagram and Lumsden's is the optical depth range in the sample of $\mathrm{OH} / \mathrm{IR}$ objects. Our sample, which is the same as used by Sevenster (2002a,b), contains a large number of $\mathrm{OH} / \mathrm{IR}$ objects sharing the same space in the colour-colour diagram with planetary nebulae and transition objects. These $\mathrm{OH} / \mathrm{IR}$ objects are about to leave the AGB branch, as we discuss in the next section and as has been reported by Sevenster $(2002 a, b)$ and they are missing in the work of Lumsden.

\section{Classification of very red $\mathrm{OH} / \mathrm{IR}$ objects}

In Sect. 3 we identified a population of $\mathrm{OH} / \mathrm{IR}$ objects with high colour indices that are separated from the main bulk of $\mathrm{OH} / \mathrm{IR}$ objects by a gap (Fig. 3). Hereafter those objects will be referred as "very red OH/IR objects" (VR-OH/IR objects). Sevenster (2002a) used MSX and IRAS colour indices to study her sample of $\mathrm{OH} / \mathrm{IR}$ objects and found a similar sub-sample of very red $\mathrm{OH} / \mathrm{IR}$ objects in her [8]-[12] $\times$ [15]-[21] colour-colour diagram. A comparison between that diagram and Fig. 3 of the present work reveals that the VR-OH/IR objects correspond mostly to the OH/IR objects in quadrants I and IV of Sevenster. This correspondence is straightforward because the colours [8]-[12] and [8.3]-[14.7] are quite similar. Sevenster also carried out a study of the $\mathrm{OH}$ spectra of the various types of $\mathrm{OH} / \mathrm{IR}$ object, concerning their position in MSX and IRAS colour-colour diagrams. She points out that the majority (61 to $80 \%$ ) of the $\mathrm{OH} / \mathrm{IR}$ objects are double-peaked, and the fraction of $\mathrm{OH} / \mathrm{IR}$ objects that show single-peak or irregular $\mathrm{OH}$ spectra does not change significantly with the mid-infrared colours. On the other hand, the expansion velocity of the $\mathrm{OH}$ envelope is related to the [8]-[12] colour index in the sense that the VR-OH/IR objects rarely show expansion velocities greater than $15 \mathrm{~km} \mathrm{~s}^{-1}$. Since some of these objects might be post-AGB stars, she concludes also that the outflow velocities of the $\mathrm{OH}$ envelope of post-AGB stars are lower than $15 \mathrm{~km} \mathrm{~s}^{-1}$. In spite of the conclusions drawn above, the nature of the members of the group of VR-OH/IR stars remained "possibly post-AGB" or "late-AGB oxygen-rich stars", whilst the relative contribution of the various classes is still undetermined. The aim of this section is to present the results of a survey in the literature intended to establish the nature of the VR-OH/IR objects.

The VR-OH/IR objects constitute a mixed population, which includes objects that are still in the AGB (the $\mathrm{OH} / \mathrm{IR}$ stars with very high mass-loss rates), sources that have already left this stage (transition objects, planetary nebulae), and young objects (protostars, molecular clouds, etc.). We used the SIMBAD database to find literature on all 49 VR-OH/IR objects. For each object we examined the original references for a classification other than "OH/IR object" or "infrared source". Objects classified in the literature as "protoplanetary nebula" and "post-AGB star" have been brought together under the designation "transition objects". We have kept the original term "possible PN" for a possible planetary nebula and "HII" for a HII region, which might be interpreted as an $\mathrm{OH} / \mathrm{IR}$ object of interstellar origin near a HII region. We emphasize that we did not classify the objects, but simply compiled the classifications given in the original references. When a source did not have a classification, it was considered as "OH source".

In addition to the classification described above, we examined visually the $49 \mathrm{OH}$ spectra and classified them according to the following criteria:

1. $2 \mathrm{P}=$ double peak;

2. $2 \mathrm{~Pa} 1=$ asymmetric double peak, low velocity weaker;

3. $2 \mathrm{~Pa} 2=$ asymmetric double peak, high velocity weaker;

4. $1 \mathrm{P}=$ single peak;

5. $\mathrm{B}=$ bump emission (one single, broad peak).

We also include the $\mathrm{L}$ classification that can be added to the types above for a broad line (except the B type). We consider a broad feature (L) the cases where the full width at half maximum $(F W H M)$ in units of $\mathrm{km} \mathrm{s}^{-1}$ is in the range $15<F W H M<25$ and a bump (B) when $F W H M>25$. 
Table 5. Very red OH/IR sources; the $\mathrm{OH}$ type is defined in the text. The classification is given as in the original reference. The $\mathrm{OH}$ references are: $\mathrm{A}=$ Sevenster et al. (1997a); B = Sevenster et al. (1997b); and C = Sevenster et al. (2001). The references between brackets are: (a) = Nyman et al. (1998); (b) = van den Steene et al. (2000); (c) = Hrivnak et al. (1999); (d) = Preite-Martinez (1988); (e) = Gomez et al. (1990); (f) = Deguchi et al. (2000); (g) = Garcia-Lario et al. (1997); (h) = Fish et al. (2003); (i) = Engels (2002); (j) = Zijlstra et al. (1989); (k) = Likkel et al. (1991); (l) = Likkel (1989); (m) = Kohoutek (2002); (n) = Walsh et al. (1998); (o) = Sevenster (2002b); (p) = te Lintel Hekkert et al. (1989); (q) = Zijstra et al. (2001); (r) = Blommaert et al. (1994); (s) = Lewis et al. (1990); and (t) = Fix \& Mutel (1984).

\begin{tabular}{|c|c|c|c|c|c|}
\hline $\mathrm{OH}$ name & OH type & OH Ref. & Classification & other masers & Refs. \\
\hline $\mathrm{OH} 314.933-02.052$ & $2 \mathrm{Pal}$ & $\mathrm{B}$ & Possible PN & $\mathrm{SiO}$ & (a) \\
\hline OH 323.459-00.079 & $1 \mathrm{P}$ & B & HII region & & (n) \\
\hline OH $326.932+02.754$ & $2 \mathrm{P}$ & B & $\mathrm{OH}$ source & & \\
\hline OH 326.530-00.419 & 2Pa1-L & B & OH source & & \\
\hline OH $335.832+01.434$ & $2 \mathrm{P}$ & B & Post-AGB Star & & $(b, o)$ \\
\hline OH $337.065-01.173$ & $1 \mathrm{P}$ & B & $\mathrm{OH}$ source & & \\
\hline OH $353.844+02.984$ & $1 \mathrm{P}$ & A & "Cotton Candy" - Post-AGB & & \\
\hline OH $353.973+02.727$ & $2 \mathrm{~Pa} 2$ & B & PN (GLMP 546) & & $(\mathrm{m}, \mathrm{o})$ \\
\hline OH 348.813-02.840 & 2Pa1 & B & "Walnut Nebulae" - Post-AGB & & (c) \\
\hline OH 353.945-00.972 & 2Pa1 & A & Post-AGB Star & & $(\mathrm{d}, \mathrm{o})$ \\
\hline OH $359.140+01.137$ & 2Pa1 & A & Post-AGB Star & $\mathrm{H}_{2} \mathrm{O}, \mathrm{SiO}$ & $(e, o)$ \\
\hline $\mathrm{OH} 002.348+02.965$ & $2 \mathrm{P}$ & A & $\mathrm{OH} / \mathrm{IR}$ Star & $\mathrm{SiO}$ & (f) \\
\hline OH 357.092-00.362 & $2 \mathrm{~Pa} 2$ & A & $\mathrm{OH} / \mathrm{IR}$ Star & & (p) \\
\hline $\mathrm{OH} 000.207+01.414$ & 1P-L & A & IRAS $17375-2759 \mathrm{PN}$ & $\mathrm{SiO}$ & (a) \\
\hline OH 359.233-01.876 & $2 \mathrm{P}$ & A & Post-AGB Star & & $(\mathrm{g}, \mathrm{o})$ \\
\hline $\mathrm{OH}$ 002.286-01.801 & 2Pa1 & A & Post-AGB Star & & $(\mathrm{g}, \mathrm{o})$ \\
\hline OH 005.885-00.392 & $1 \mathrm{P}$ & $\mathrm{C}$ & HII region & & (h) \\
\hline OH $003.471-01.853$ & $\mathrm{~B}$ & A & IRAS 17576-2653 - Possible PN & & (d) \\
\hline OH 004.017-01.679 & $2 \mathrm{P}$ & A & Post-AGB Star & & $(\mathrm{g}, \mathrm{o})$ \\
\hline OH $011.560+00.088$ & 2Pa1 & $\mathrm{C}$ & $\mathrm{OH} / \mathrm{IR}$ Star & & (j) \\
\hline ОH 015.364+01.925 & $2 \mathrm{~Pa} 2$ & $\mathrm{C}$ & Post-AGB Star & & $(g, o)$ \\
\hline OH 016.240-00.622 & 2Pa1 & $\mathrm{C}$ & $\mathrm{OH}$ source & & \\
\hline $\mathrm{OH} 020.854+00.486$ & $\mathrm{~B}$ & $\mathrm{C}$ & $\mathrm{OH} / \mathrm{IR}$ Star & & $(p, q)$ \\
\hline OH 017.684-02.032 & $2 \mathrm{P}$ & $\mathrm{C}$ & Post-AGB Star & $\mathrm{H}_{2} \mathrm{O}$ & $(\mathrm{i}, \mathrm{o})$ \\
\hline $\mathrm{OH} 023.751+00.210$ & 2Pa1 & $\mathrm{C}$ & $\mathrm{OH} / \mathrm{IR}$ Star & & (j) \\
\hline $\mathrm{OH} 025.154+00.060$ & $2 \mathrm{P}$ & $\mathrm{C}$ & $\mathrm{OH} / \mathrm{IR}$ Star & & (r) \\
\hline OH 026.063-00.519 & 2Pa1 & $\mathrm{C}$ & $\mathrm{OH}$ source & & \\
\hline OH 027.577-00.853 & $2 \mathrm{P}$ & $\mathrm{C}$ & Post-AGB Star & & $(g, o)$ \\
\hline $\mathrm{OH} 038.909+03.178$ & 2Pa1 & $\mathrm{C}$ & Post-AGB Star & & $(\mathrm{g}, \mathrm{o})$ \\
\hline $\mathrm{OH} 038.287+01.875$ & 2Pa1 & $\mathrm{C}$ & $\mathrm{OH} / \mathrm{IR}$ Star & & (s) \\
\hline OH 035.209-02.653 & $2 \mathrm{P}$ & $\mathrm{C}$ & GLMP 870 - Post-AGB Star & $\mathrm{H}_{2} \mathrm{O}$ & $(\mathrm{g}, \mathrm{k}, \mathrm{l})$ \\
\hline OH $043.165-00.028$ & $1 \mathrm{P}$ & $\mathrm{C}$ & $\mathrm{OH}$ source & & \\
\hline OH $341.275-00.720$ & $2 \mathrm{~Pa} 2$ & B & $\mathrm{OH}$ source & & \\
\hline OH 345.052-01.855 & 2Pa1 & B & $\mathrm{OH}$ source & & \\
\hline OH 348.668-00.715 & $2 \mathrm{~Pa} 2$ & B & $\mathrm{OH}$ source & & \\
\hline OH $349.804-00.321$ & $2 \mathrm{~Pa} 2$ & B & Post-AGB Star & & $(\mathrm{g}, \mathrm{o})$ \\
\hline OH 351.474-00.596 & $2 \mathrm{~Pa} 2$ & A & $\mathrm{OH}$ source & & \\
\hline $\mathrm{OH} 353.637+00.815$ & $2 \mathrm{~Pa} 2$ & A & Post-AGB Star & & (o) \\
\hline ОH $000.260+01.027$ & $2 \mathrm{~Pa} 2$ & A & OH source & & \\
\hline OH 001.484-00.061 & 2Pa1 & A & $\mathrm{OH} / \mathrm{IR}$ Star & & (p) \\
\hline $\mathrm{OH} 007.961+01.445$ & $2 \mathrm{P}$ & $\mathrm{C}$ & Post-AGB Star & & $(\mathrm{g}, \mathrm{o})$ \\
\hline OH 009.097-00.392 & $1 \mathrm{P}$ & $\mathrm{C}$ & $\mathrm{OH}$ source & & \\
\hline OH 006.594-02.011 & $2 \mathrm{~Pa} 2$ & $\mathrm{C}$ & Post-AGB Star & & (o) \\
\hline OH 007.420-02.042 & 2Pa1 & $\mathrm{C}$ & $\mathrm{OH}$ source & & \\
\hline $\mathrm{OH} 015.700+00.770$ & $2 \mathrm{~Pa} 2$ & $\mathrm{C}$ & Post-AGB Star & $\mathrm{H}_{2} \mathrm{O}$ & $(i, o)$ \\
\hline OH 022.043-00.608 & $2 \mathrm{~Pa} 2$ & $\mathrm{C}$ & $\mathrm{OH} / \mathrm{IR}$ Star & & (t) \\
\hline $\mathrm{OH} 030.944+00.035$ & $1 \mathrm{P}$ & $\mathrm{C}$ & $\mathrm{OH}$ source & & \\
\hline OH 030.394-00.706 & $1 \mathrm{P}$ & $\mathrm{C}$ & Post-AGB Star & & (o) \\
\hline OH $037.118-00.847$ & $2 \mathrm{~Pa} 2$ & $\mathrm{C}$ & Post-AGB Star & $\mathrm{H}_{2} \mathrm{O}$ & $(i, o)$ \\
\hline
\end{tabular}


Table 5 shows the $\mathrm{OH}$ names of the VR-OH/IR sources, the type of $\mathrm{OH}$ spectrum, and its classification according to the original references given in the SIMBAD database. Among the $49 \mathrm{VR}-\mathrm{OH} / \mathrm{IR}$ objects, 2 (4\%) are classified as planetary nebulae, $2(4 \%)$ are cited as possible planetary nebulae, $20(41 \%)$ as transition objects, $9(18 \%)$ as $\mathrm{OH} / \mathrm{IR}$ stars, $14(29 \%)$ as $\mathrm{OH}$ sources (i.e. without classification), and 2 objects (4\%) are associated with HII regions. The three former categories (49\% of the objects) comprise objects that have already left the AGB, but this figure might be even larger, since some of the 14 objects cited as $\mathrm{OH}$ sources lack a more detailed examination and might be in the post-AGB evolutionary phase. Finally, a minor part of the objects is believed to be related to star forming regions, which is in agreement with the results of Felli et al. (2000), who identified young stellar objects in the ISOGAL survey with similar colours $([7]-[15]>2.5)$ in a field near $l=+45^{\circ}$.

An examination of the $\mathrm{OH}$ types of the various classes of object in Table 5 confirms the statement by Sevenster (2002a) that transition objects can be found with different kinds of $\mathrm{OH}$ spectra, i.e. the $\mathrm{OH}$ spectrum alone does not determine whether an object has left the AGB or not. In fact, the transition objects cited in the literature might have double-peak (18 objects) or single-peak (2 objects) OH spectra.

\section{Theoretical tracks beyond the AGB phase}

In this section, we compare the colour-colour diagrams described in the previous section with theoretical predictions of magnitudes and colours. The model for post-AGB evolution assumed in this work was proposed in Volk (1993) and is shortly described here.

Each model starts when the star leaves the AGB i.e., its envelope becomes detached from the core and is driven away at the speed of $10 \mathrm{~km} \mathrm{~s}^{-1}$. The evolution of the core is that calculated by Schönberner $(1981,1983)$ for $0.64 M_{\odot}$, but accelerated by a factor 2 in order to match the IRAS colours of planetary nebulae. It is well known that the evolution time in the postAGB phase depends on the mass-loss rate during the AGB, and authors have found a wide range of times, depending on the mass-loss rate assumed. Another hypothesis assumed in the models used is that the star leaves the AGB burning hydrogen, i.e. between two thermal pulses. According to Iben (1984), post-AGB evolution is dominated by helium burning if the star leaves the AGB in the phase range $0<\phi<0.15$, where the phase $\phi=0$ at the time of the beginning of the thermal pulse. Post-AGB stars might also have a late pulse during the horizontal evolution across the HR diagram, if the star leaves the AGB near to having a pulse, let us say for $\phi>0.9$, approximately. During the post-AGB helium burning the star loops in the HR diagram and returns to the AGB in about 200 years (Blöcker 1995). Considering the figures above, we can state that helium burning applies in the minority of the cases. As a result, the effects of thermal pulses for stellar evolution in colour-colour diagrams are presently unknown and difficult to predict.

The circumstellar dust shell and the neutral gas are modelled following Spagna \& Leung (1983). The models end

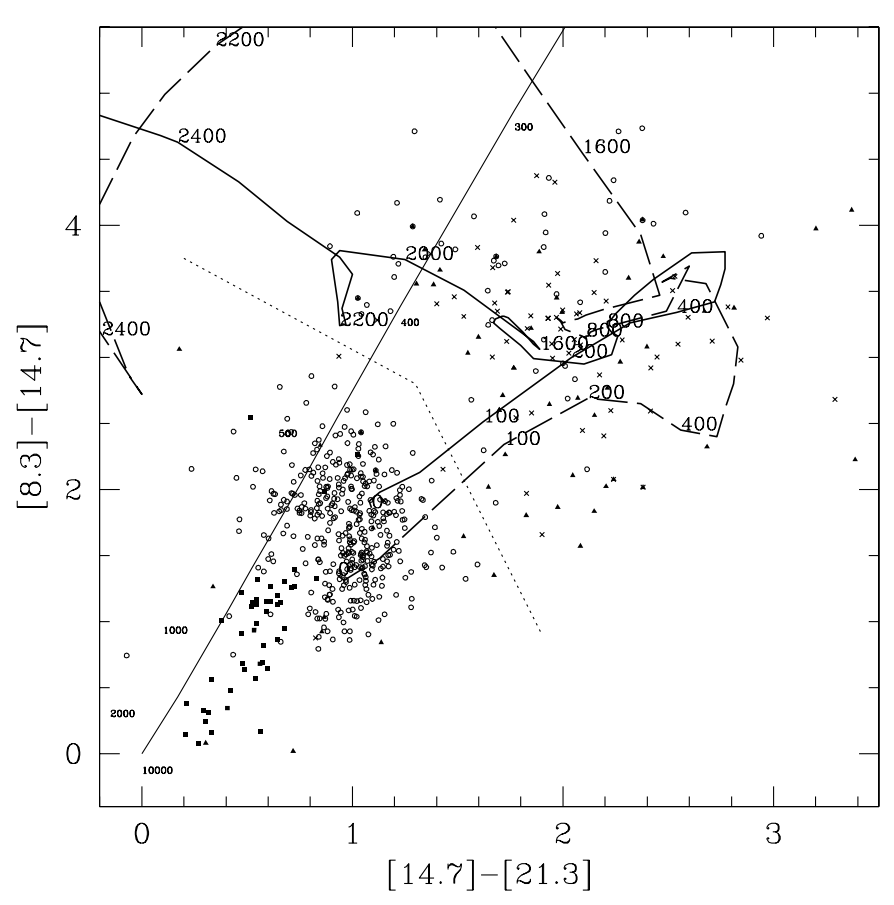

Fig. 4. Evolutionary tracks of an oxygen-rich star in the post-AGB phase. Evolution time is plotted next to the curves and is counted in years. The two curves correspond respectively to mass loss rates of $2.1 \times 10^{-5} M_{\odot} /$ year (model a, continuous line) and $5.2 \times$ $10^{-6} M_{\odot} /$ year (model $\mathrm{b}$, dashed line). The symbols are the same as in Figs. 2 and 3 but have been shrunk to make the curves clearer.

at 10000 years of evolution, when numerous emission lines dominate the spectrum together with a strong infrared continuum in the mid-infrared. The mass-loss rates at the end of the AGB are $2.1 \times 10^{-5} M_{\odot} /$ year for models $a$ and $c$ and $5.2 \times 10^{-6} M_{\odot} /$ year for models $b$ and $d$, respectively. We take four different sets of parameters for the circumstellar dust shell in Volk's model: (a) silicates, initial optical depth 10.0 at $9.7 \mu \mathrm{m}$; (b) silicates, initial optical depth 2.5 at $9.7 \mu \mathrm{m}$; (c) graphite, initial optical depth 2.5 at $11.22 \mu \mathrm{m}$; (d) graphite, initial optical depth 0.625 at $11.22 \mu \mathrm{m}$. Each set of spectra (two silicate and two graphite) was convolved with the MSX filter curves given in the MSX Explanatory Guide (Egan et al. 1999). We do not consider colour-colour diagrams that include the $K$ band because of limitations in the program that cause this magnitude to be inaccurate.

Figures 4 and 5 reproduce the [14.7]-[21.3] $\times[8.3]-[14.7]$ described in the previous section together with the evolutionary tracks described above. As one can see in the figures, the four evolutionary tracks start at the crowd of points that represent AGB stars with high mass-loss rates. The time the star spends together with the AGB stars is extremely short: less than 100 and 300 years for oxygen and carbon-rich stars, respectively. This short time accounts for the gap that separates the VR-OH/IR objects, planetary nebulae, and transition objects, from the bluer group of $\mathrm{OH} / \mathrm{IR}$ objects (dotted line in Figs. 3-5). Eventually the stars evolve rapidly towards the upper-right part of the diagram, into the region where transition objects, planetary nebulae, and VR-OH/IR objects are located. 


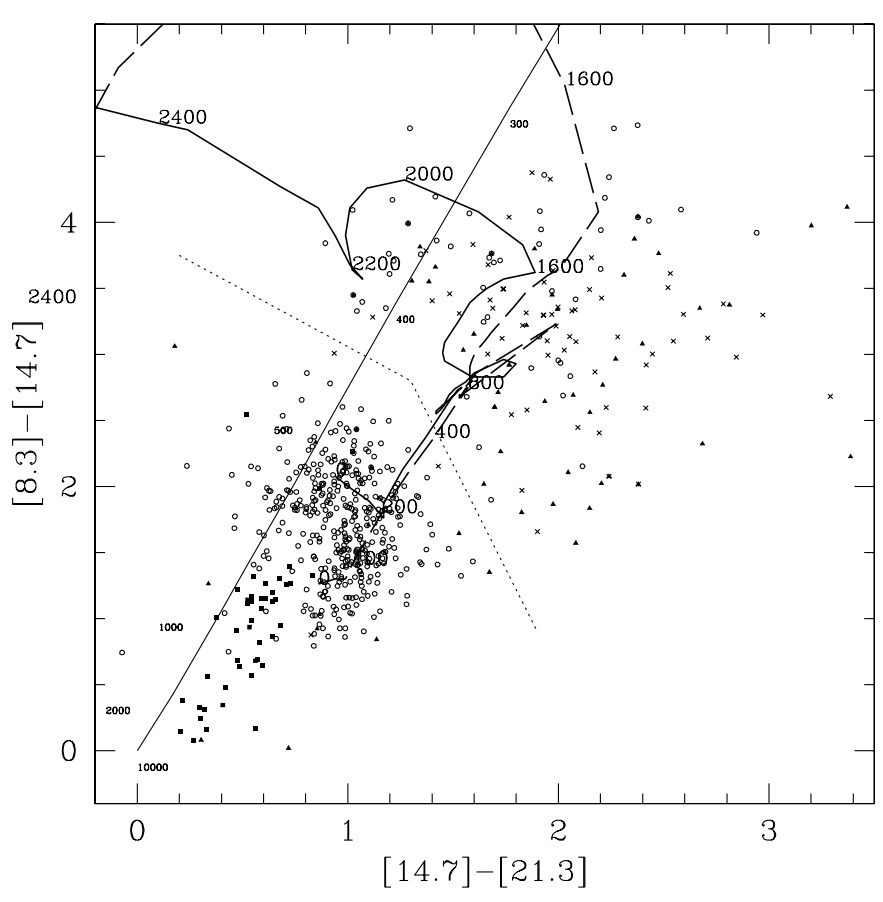

Fig. 5. The same as Fig. 4, but for carbon-rich stars with massloss rates of $2.1 \times 10^{-5} M_{\odot} /$ year (model c, continuous line) and $5.2 \times 10^{-6} M_{\odot} /$ year (model d, dashed line). Symbols used are the same as in Figs. 2 and 3.

In this region the tracks are quite clumsy but they cover the cloud of points that are spread over a wide range of colours. Here some differences between oxygen- and carbon-rich objects should be noted: according to the models, O-rich objects can have a redder colour after 400 years of post-AGB evolution, whilst the carbon-rich ones are more restricted in colour and are not expected to be redder than [14.7]-[21.3] $=2.2$. Eventually the star will leave the region where transition objects and $\mathrm{PNe}$ are, and head for the upper-left region of the diagram in a time that will strongly depend on the parameters of the model: 2200 years for models $a$ and $c$ (that have higher mass-loss rates), and 1600 years for models $b$ and $d$, which corresponds to core temperatures of $1.9 \times 10^{5} \mathrm{~K}$ and $1.1 \times 10^{5} \mathrm{~K}$, respectively. After that point, models and data disagree completely. The diagrams show that transition objects and planetary nebulae should have similar colours but the models predict that planetary nebulae evolve differently, first having high [8.3]-[14.7] colour index and eventually having negative [14.7]-[21.3] colour index. Our sample contains planetary nebulae in various evolutionary stages, but there are no PNe with colours that match the predictions of the models after 2200 years of evolution, therefore we can conclude that they do not work correctly after that point. Volk (1992) used another grid of models to study planetary nebulae in the IRAS [12]-[25] $\times[25]-[60]$ colour-colour diagram and also in his diagrams the evolutionary tracks reproduce well the colours of $\mathrm{PNe}$, but they end up quite far from the main concentration of points at $t \simeq 7 \times 10^{3}$ years of evolution. These differences of $t$ between the various models occur because how quickly a post-AGB evolves depends mainly on the mass-loss rate assumed in the AGB and this has been chosen to match the observations. Apart from these discrepancies, the models show that the wide range of colours observed is not caused by variability or bad measurements, although they can play a role, but is the result of normal evolution during the post-AGB phase. The models also explain why there is a gap between the crowd of points representing late-AGB stars and transition objects: stars cross that gap very rapidly, in two hundred years or less.

Sevenster (2002b) presents evolutionary tracks on colour-colour diagrams for the three IRAS bands and the $K$ band, based on the models proposed by van Hoof et al. (1997). Those models do not provide MSX magnitudes and colours, therefore they cannot be quantitatively compared with our results. Nevertheless there is a feature that is remarkable in their model, but is absent in ours: the red loop at the end of the AGB phase, which causes the $R_{21}$ colour to loop back towards the blue, before the star resumes its normal evolution to the red. Evolved objects are scarcely found along the blue part of those tracks, but there is a significant group of objects along the red part. The models provided by Volk used in this work do not predict red loops, and they seem to fit the data better. According to the present model, evolution towards blue colours is predicted to happen only after about $2 \times 10^{3}$ years of post-AGB evolution (see Figs. 4 and 5), when the star is halfway to the planetary nebula phase.

\section{Conclusions}

We compiled a sample containing various classes of evolved objects, such as: carbon-stars, OH/IR objects, transition (post-AGB) objects, and planetary nebulae. We searched for mid and near-infrared counterparts in the MSX survey and in the literature, and constructed colour-colour diagrams to study the evolution of these objects. We found that oxygen-rich, AGB stars $(\mathrm{OH} / \mathrm{IR})$ can be distinguished from carbon-rich stars in the $(K-[8.3]) \times([8.3]-[14.7])$ colour-colour diagram, similarly to what was proposed before by Epchtein et al. (1987). We explain the separation between the two groups of stars as the impact of the $\mathrm{SiO} 9.7 \mu \mathrm{m}$ feature on the [8.3] MSX band, that shifts $\mathrm{OH} / \mathrm{IR}$ stars away from the blackbody line. Transition objects, high mass-loss OH/IR objects, and planetary nebulae occupy the same region in this colour-colour diagram and in the $([14.7]-[21.3]) \times([8.3]-[14.7])$ diagram as well. We confirm Sevenster's (2002a) previous finding that the majority of $\mathrm{OH}$ spectra of transition objects are normal, doublepeaked. Nevertheless, among the 49 objects that show evidence of high mass-loss rate, a minor but significant percentage $(22 \%)$ has $\mathrm{OH}$ spectra that differ from the classic double peak, perhaps because of some mechanism related to the superwind phenomenon. We tested a grid of four models with different $\mathrm{C} / \mathrm{O}$ ratios, mass-loss rates and optical depths developed by Volk (1992, 1993). A comparison between the infrared colours indices predicted by the model and the observations showed that the gap that separates objects with optically thick envelopes from those with small optical depth values is caused by the rapid evolution just after the star leaves the AGB. Eventually, the objects describe a tortuous trajectory in MSX colour-colour diagrams, which explains the wide range of colour indices of transition objects and planetary 
nebulae. The models reproduce well the infrared MSX colours up to 2000 years after the end of the AGB phase, i.e. when the temperature of the core reaches $1-2 \times 10^{5} \mathrm{~K}$.

Acknowledgements. We are indebted to Kevin Volk for providing us his models of post-AGB evolution. We thank the anonymous referee for his valuable suggestions and critical reading of the manuscript. This publication makes use of data products from the Two Micron All Sky Survey, which is a joint project of the University of Massachusetts and the Infrared Processing and Analysis Center, funded by the National Aeronautics and Space Administration and the National Science Foundation. This research has made use of the SIMBAD database, operated at CDS, Strasbourg, France. This work was supported by FAPESP (Fundação de Amparo à Pesquisa do Estado de São Paulo, Brazil) under grant number 2002/08816-5, and $\mathrm{CNPq}$ (Conselho Nacional de Desenvolvimento Científico e Tecnológico, Brazil). S.L.M. and W.J.M. acknowledge CNPq (processes 478309/01-5 and 3001072003-0) for financial support.

\section{References}

Acker, A., Ochsenbein, F., Stenholm, B., et al. 1992, ESO-Strasbourg Catalogue of Galactic Planetary Nebulae, Munich (ESO)

Benson, P. J., Little-Marenin, I. R., Woods, T. C., et al. 1990, ApJS, 74,911

Blöcker, T. 1995, A\&A, 299, 755

Blommaert, J. A. D. L., van Langevelde, H. J., \& Michiels, W. F. P. 1994, A\&A, 287, 479

Cox, A. N. 2000, in Allen's Astrophysical Quantities (Springer)

Deguchi, S., Fujii, T., Izumiura, H., et al. 2000, ApJS, 128, 571 (f)

Egan, M. P., Price, S. D., Shipman, R. F., \& Tedesco, E. 1997, A\&AS, 29, 1294

Egan, M. P., Price, S. D., Moshir, M. M., et al. 1999, The Midcourse Space Experiment Point Source Catalog Version 1.2 Explanatory Guide, AFGL Tech. Rep. AFRL-VS-TR-1999-1522

Engels, D. 2002, A\&A, 388, 252 (i)

Epchtein, N., Le Bertre, T., Lépine, J. R. D., et al. 1987, A\&AS, 71, 39

Epchtein, N., Le Bertre, T., \& Lépine, J. R. D. 1990, A\&A, 227, 82

Felli, M., Comoretto, G., Testi, L., Omont, A., \& Schuller, F. 2000, A\&A, 362, 199

Fish, V. L., Reid, M. J., Wilner, D. J., \& Churchwell, E. 2003, ApJ, $587,701(\mathrm{~h})$

Fix, J. D., \& Mutel, R. L. 1984, AJ, 89, 406

Garcia-Lario, P., Manchado, A., Pych, W., \& Pottasch, S. R. 1997, A\&AS, 126, $479(\mathrm{~g})$

Gezari, D. Y., Schmidt, M., \& Mead, J. M. 1987, Catalog of infrared observations, NASA reference publication No. 1196

Gomez, Y., Moran, J. M., \& Rodriguez, L. F. 1990, Rev. Mex. Astron. Astrofis., 20, 55 (e)

Guglielmo, F., Epchtein, N., Le Bertre, T., et al. 1993, A\&AS, 99, 31

Guglielmo, F., Epchtein, N., Arditti, F., \& Sevre, F. 1997, A\&AS, 122, 489

Guglielmo, F., Le Bertre, T., \& Epchtein, N. 1998, A\&A, 334, 609

Hrivnak, B. J., Kwok, S., \& Su, K. Y. L. 1999, ApJ, 524, 849 (c)

Hu, J. Y., Slijkhuis, S., de Jong, T., \& Jiang, B. W. 1993, A\&AS, 100, 413

Iben Jr., I. 1984, ApJ, 277, 333
Iben Jr., I. 1995, Phys. Rep., 250, 1

Kimeswenger, S. 2001, Rev. Mex. Astron. Astrofis., 37, 115

Kohoutek, L. 2002, AN, 323, 57 (m)

Lépine, J. R. D., Ortiz, R., \& Epchtein, N. 1995, A\&A, 299, 453

Lewis, B. M., Chengalur, J. N., Schmelz, J., \& Terzian Y. 1990, MNRAS, 246, 523

Li, A., \& Greenberg, J. M. 1997, A\&A, 323, 566

Likkel, L. 1989, ApJ, 344, 350 (1)

Likkel, L., Forveille, T., Omont, A., \& Morris, M. 1991, A\&A, 246, $153(\mathrm{k})$

Lindqvist, M., Winnberg, A., Habing, H. J., \& Matthews, H. E. 1992, A\&AS, 92, 43

Loup, C., Forveille, T., Omont, A., \& Paul, J. F. 1993, A\&AS, 99, 291

Luck, R. E., \& Webfer, G. G. 1995, AJ, 110, 2425

Lumsden, S. L., Hoare, M. G., Oudmaijer, R. D., \& Richards, D. 2002, MNRAS, 336, 621

Nyman, L.-A., Hall, P. J., \& Olofsson, H. 1998, A\&AS, 127, 185 (a)

Oudmaijer, R. D., van der Veen, W. E. C. J., Waters, L. B. F. M., et al. 1992, A\&AS, 96, 625

Pena, M., Medina, S., \& Stasinska, G. 2003, RMxAC, 18, 84

Preite-Martinez, A. 1988, A\&AS, 76, 317 (d)

Schönberner, D. 1981, A\&A, 103, 119

Schönberner, D. 1983, ApJ, 272, 708

Sevenster, M. N., Chapman, J. M., Habing, H. J., Killeen, N. E. B., \& Lindqvist, M. 1997a, A\&ASS, 122, 79 (A)

Sevenster, M. N., Chapman, J. M., Habing, H. J., Killeen, N. E. B., \& Lindqvist, M. 1997b, A\&AS, 124, 509 (B)

Sevenster, M., van Langevelde, H. J., Moody, R. A., et al. 2001, A\&A, 366, $481(\mathrm{C})$

Sevenster, M. 2002a, AJ, 123, 2772

Sevenster, M. 2002b, AJ, 123, 2788 (o)

Sjouwerman, L. O., van Langevelde, H. J., Winnberg, A., \& Habing, H. J. 1998, A\&AS, 128, 35

Skrutskie, et al. 1997, in The Impact of Large Scale Near-IR Sky Surveys, ed. F. Garzon et al. (Netherlands: Kluwer), 25

Spagna, G. F., \& Leung, C. M. 1983, Comput. Phys. Comm., 28, 337

te Lintel Hekkert, P., Versteege-Hensel, H. A., Habing, H. J., \& Wiertz, M. 1989, A\&AS, 78, 399

van den Steene, G. C., van Hoof, P. A. M., \& Wood, P. R. 2000, A\&A, 362, 984 (b)

van der Veen, W., \& Habing, H. J. 1988, A\&A, 194, 125

van Hoof, P., Oudmaijer, R., \& Waters, L. 1997, MNRAS, 289, 371

van Loon, J. T., Zijlstra, A. A., Whitelock, P. A., et al. 1997, A\&A, 325,585

van Loon, J. Th., Zijlstra, A. A., Whitelock, P. A., et al. 1998, A\&A, 329, 169

Vijapurkar, J., Parthasarathy, M., \& Drilling, J. S. 1998, B.A.S.I., 26, 497

Volk, K. 1992, ApJS, 80, 347

Volk, K. 1993, in Planetary Nebulae, ed. R. Weinberger, \& A. Acker (Dordrecht: Kluwer), IAU Symp., 155, 469

Walsh, A. J., Burton, M. G., Hyland, A. R., \& Robinson, G. 1998 , MNRAS, 301, 640 (n)

Zijlstra, A. A., te Lintel Hekkert, P., Pottasch, S. R., et al. 1989, A\&A, $217,157(\mathrm{j})$

Zijlstra, A. A., Chapman, J. M., te Lintel Hekkert, P., et al. 2001, MNRAS, 322, 280 